\title{
Morbilidad Postoperatoria por Colangiografía Endoscópica Retrógrada con o sin Papilotomía Ulterior. Serie de Casos
}

\author{
Postoperative Morbidity by Endoscopic Retrograde Cholangiography \\ with or without Ulterior Papillotomy. Series of Cases
}

\author{
Julio Figueroa ${ }^{*, * *, * * *, * * * *}$; Carlos Manterola ${ }^{* * *, * * * * *, * * * * * *}$; Eric Castillo ${ }^{*, *, * * * ;}$ \\ Camila Figueroa**; Renato Carrasco, ${ }^{* * * * *}$ \& John Bohle, ${ }^{* * * * * *}$
}

FigueroA, J.; MANTEROla, C.; CASTIllo, E.; FIGUEROA, C.; CARRASCO, R. \& BOHLE, J. Morbilidad postoperatoria por colangiografía endoscópica retrógrada con o sin papilotomía ulterior. Serie de casos. Int. J. Morphol., 33(2):566-570, 2015.

RESUMEN: La patología biliar litiásica es frecuente en nuestro país, con prevalencias entre 30\% y 50\%; y la ictericia obstructiva secundaria a coledocolitiasis (IOC), constituye un motivo de consulta frecuente en los servicios de urgencia. Por otra parte, la papilotomía endoscópica (PE) post colangiografía retrógrada endoscópica (CRE), constituye el tratamiento de elección en estos casos; sin embargo, es un procedimiento no exento de complicaciones. El objetivo de este estudio, es describir la MPO e identificar posibles factores de riesgo (FR) asociados a MPO, en pacientes con IOC, sometidos a PE. Serie de casos retrospectiva, de pacientes con IOC, a quienes se les realizó CRE y ulterior PE. La variable resultado fue desarrollo de MPO (hemorragia, perforación y pancreatitis). Otras variables de interés fueron canulación, dificultad de ésta, desarrollo de PE, uso de pre corte y mortalidad. La recolección de datos se realizó mediante una pauta ad-hoc, en la que se registraron las variables extraídas desde el protocolo operatorio y la ficha clínica. Se aplicó estadística descriptiva y analítica (Chi2 de Pearson y exacto de Fisher) para estimar fuerza de asociación. Se intervinieron 200 pacientes. La Media de edad fue de $60 \pm 18$ años; $62 \%$ eran mujeres $(n=124)$. Se registró MPO en 32 casos $(16,0 \%)$ : Perforación $(0,5 \%)$, pancreatitis $(2,0 \%)$ y hemorragia $(13,5 \%)$. La serie no registró mortalidad. No se logró objetivar asociación entre la variable "canulación difícil" y las variables hemorragia $(\mathrm{p}=0,214)$; pancreatitis $(\mathrm{p}=0,519)$; ni perforación $(\mathrm{p}=1)$. Sin embargo, se verificó asociación entre el desarrollo de hemorragia y la realización de PE $(\mathrm{p}=0,017)$. La hemorragia es la MPO más frecuente en esta serie; y la PE es un FR para el desarrollo de hemorragia.

PALABRAS CLAVE: Coledocolitiasis; Litiasis de la vía biliar; Colangiografía retrógrada endoscópica; Cirugía de la coledocolitiasis; Papilotomía endoscópica; Cirugía endoscópica.

\section{INTRODUCCIÓN}

La patología biliar litiásica es entidad nosológica frecuente en nuestro país, alcanzando cifras de prevalencias reportadas entre $30 \%$ y $50 \%$. Por otra parte, la ictericia secundaria a coledocolitiasis (IOC), constituye un motivo frecuente de consulta en los servicios de urgencia (ASGE Standards of Practice Committee et al., 2012).

El diagnóstico ideal es a través de colangio resonancia nuclear magnética (Verma et al., 2006; Mandelia et al., 2013); el que luego se confirma mediante colangiografía retrógrada endoscópica (CRE) (Güitrón-Cantú et al., 2002); procedimiento habitual, que permite la visualización del sistema biliar, mediante la canulación de la papila de Vater y la inyección retrógrada de material de contraste, haciendo uso de un duodenoscopio de visión lateral.

A pesar que la cirugía convencional se sigue practicando de forma rutinaria en los servcios públicos (en especial de hospitales pequeños), la papilotomía endoscópica (PE), constituye el tratamiento de elección en estos casos; sin embargo, es un procedimiento invasivo no exento de complicaciones graves (ASGE Standards of Practice

\footnotetext{
Servicio de Cirugía, Hospital de Puerto Montt, Puerto Montt, Chile.

** Universidad San Sebastián, Puerto Montt, Chile.

**** Programa de Magíster en Ciencias, mención Cirugía, Universidad de La Frontera, Temuco, Chile.

**** Unidad de Endoscopia Terapéutica, Hospital de Puerto Montt, Puerto Montt, Chile.

****** Centro de Excelencia en Estudios Morfológicos y Quirúrgicos (CEMyQ), Universidad de La Frontera, Temuco, Chile.

******* Centro de Investigación en Ciencias Biomédicas, Universidad Autónoma de Chile, Temuco, Chile.
} 
Committee et al.; Colton \& Curran, 2009) como: pancreatitis (1,6-15\%) (Colton \& Curran; Freeman et al., 2001; Masci et al., 2003; Wang et al., 2009), perforación (0,1-0,6\%) (Colton \& Curran; Enns et al., 2002; Howard et al., 1999); y hemorragia $(1,3-1,5 \%)$ (Colton \& Curran; Ferreira \& Baron, 2007); incluso de mortalidad (0,25\%) (Espinel et al., 2007).

Sin embargo, se estima que endoscopistas que realizan más de una PE por semana respecto de quienes realizan menos procedimientos por unidad de tiempo; debieran tener una tasa de complicaciones de alrededor de $8 \%$ vs. $11 \%$; y de menor gravedad de las mismas $0,9 \%$ vs. 2,3\% (Freeman et al., 1996).

El objetivo de este estudio, es describir la MPO e identificar posibles factores de riesgo (FR) asociadoos a MPO, en pacientes con IOC, sometidos a PE.

Este manuscrito ha sido redactado siguiendo la lista de chequeo para estudios observacionales descriptivos (Manterola \& Astudillo, 2013) .

\section{MATERIAL Y MÉTODO}

\section{Tipo de estudio: Serie de casos retrospectiva.}

Población: Mediante un muestreo no probabilístico por conveniencia, se incluyeron pacientes portadores de ictericia obstructiva de origen biliar litiásico, a quienes se les realizó una CRE y posterior PE, en la Unidad de Endoscopia Terapéutica del Hospital de Puerto Montt, Chile entre el 01 Enero 2012 al 30 de Abril 2013. Se excluyeron pacientes a quienes se les practicó CRE y PE en el intraoperatorio de una colecistectomía en la que se encontró coledocolitiasis concomitante a la colecistolitiasis.

Protocolo de estudio: Se confeccionó una hoja de recogida de datos en las que se registraron las variables biodemográficas, clínicas y peroperatorias de los pacientes (extraídas desde el protocolo operatorio y la ficha clínica).

Variables de interés: La variable resultado principal fue desarrollo de morbilidad operatoria (MPO [hemorragia, perforación y pancreatitis]), la que se trabajó de forma dicotómica [no/sí], para lo que se exigió un seguimiento mínimo de 30 días). Otras variables de interés preoperatorias fueron: Otras variables de canulación, dificultad de ésta, realización de PE, uso de pre corte y mortalidad.

Protocolo de seguimiento: Se realizó control clínico entre el $7^{\circ}$ y $10^{\circ}$ día; y luego a los 30 días del postoperatorio; y de ahí en adelante según necesidades de los pacientes; con un tiempo de seguimiento mínimo de un año.

Tratamiento realizado: En la totalidad de los casos, se realizó PE ulterior a una CRE en pabellón de acuerdo a técnica clásica (canulación, paso deguía, inyección de medio de contraste, papilotomía y extracción de cálculo o inserción de prótesis); bajo anestesia general, utilizando gases o Propofol@. En todos los casos se utilizó profilaxis antibiótica con Cefazolina 1g vía intravenosa, con excepción de quien estuviese recibiendo antibióticos al momento del procedimiento; posterior al cual, los pacientes permanecieron en la sala de recuperación de anestesia por un lapso de $2 \mathrm{~h}$. Se indicó régimen 0 , hidratación y analgesia intravenosa por $24 \mathrm{~h}$, posterior a lo cual se reiniciaron alimentación oral.

Sesgos: Para reducir el sesgo de medición, un investigador independiente, distinto de los cirujanos que operaron; recolectó los datos, previamente recibir capacitación y supervisión directa en los primeros 40 registros.

Plan de análisis: Se realizó análisis exploratorio de los datos. Se utilizó estadística descriptiva con cálculo de porcentajes, medidas de tendencia central y dispersión. Para comprobar la asociación entre las variables categóricas se utilizó la prueba de la Chi2 de Pearson y exacto de Fisher. Los datos fueron analizados con un programa IBM SPSS 19.0.

Aspectos éticos: Se observaron las guías éticas para investigación en seres humanos (Declaración de Helsinki) y los datos se trataron confidencialmente.

Fuentes de financiación: El estudio no contó con fuentes formales de financiamiento.

\section{Definiciones:}

Canulación difícil: Aquella que requierió de 10 o más intentos. Si en el protocolo aparece declarado la dificultad para realizarla, o se requirió de pre corte para lograrla.

Pancreatitis post procedimiento: Cuadro de dolor epigástrico en faja que aparece posterior a procedimiento endoscópico con alza de amilasa 3 veces el valor normal o imagen sugerente en la tomografía computada o ecografía tomada $24 \mathrm{~h}$ después del procedimiento.

Hemorragia: Complicación directa de la PE. Necesidad de realizar acción terapéutica como inyección de adrenalina 1:20, coagulación con papilotomo o aguja de pre corte, uso 
de clips endoscópicos y si obliga o no a suspender el procedimiento. Descenso de 3g/dl de hemoglobina o evidencias clínicas de hemorragia digestiva hasta 2 semanas posterior al procedimiento.

Perforación: Cuadro de dolor abdominal asociado a imágenes sugerentes en la tomografía computadorizada o radiología simple de abdomen (aire retroperitoneal o subfrénico).

\section{RESULTADOS}

En el período estudiado, se trataron de esta forma, un total de 200 pacientes, con un promedio de edad de $60 \pm 18$ años; $62,0 \%$ de los cuales fueron de sexo femenino $(n=124$ casos).

El diagnóstico posterior al procedimiento más frecuente fue coledocolitiasis 69,0\% $(n=138)$.

Se realizó PE en un $68,5 \%$ de los casos $(n=137)$ debiendo realizarse pre corte con papilótomo o con aguja en un $19,5 \%$ de ellos $(n=39)$.

En un $7 \%$ de los pacientes $(\mathrm{n}=14)$, no se logró la canulación de la vía biliar lo que da un éxito de canulación de un $93,0 \%(n=186)$.

Respecto de complicaciones observadas, reportamos que del total de CRE y PE realizadas, se verificó perforación, pancreatitis y hemorragia en un caso, 4 casos y 27 casos respectivamente $(0,5 \% ; 2, \%$; $13,5 \%$ respectivamente).

No ocurrió mortalidad en esta serie.

$\mathrm{Al}$ aplicar estadística analítica para determinar asociación entre la variable en estudio "canulación difícil" y las variables relacionadas con MPO, se logró objetivar que: la hemorragia se presentó en el 7,5\% de los casos de canulación difícil y en el 15,0\% de casos de canulación fácil $(\mathrm{p}=0,214)$; que se objetivó un $2,5 \%$ de casos de pancreatitis aguda casos de canulación difícil y un $1,4 \%$ en casos de canulación fácil ( $\mathrm{p}=0,519)$; $y$, que no hubo perforaciones en los 40 casos de canulación difícil, pero hubo un $0,7 \%$ en aquellos de canulación fácil ( $\mathrm{p}=1)$. Por otra parte, al estudiar asociación entre el desarrollo de hemorragia y PE, se verififcó un 20,7\% de hemorragia en pacientes sometidos a PE vs. 3,8\% en pacientes en los que no se realizó $\mathrm{PE}$ ( $\mathrm{p}=$ 0,017); verificándose de este modo que la PE es un FR para el desarrollo de hemorragia.

\section{DISCUSIÓN}

La mayoría de los cálculos se originan en la vesícula biliar y migran al conducto biliar común, vía el cístico. Algunos cálculos llegan a formarse dentro del conducto biliar principal, con una vesícula biliar aparentemente normal, tales pacientes representan el $10 \%$ de todos los casos. Aproximadamente el $5 \%$ de los pacientes que son sometidos a una colecistectomía, tienen un cálculo residual o de neoformación (Hermann, 1989). Desde la primera canulación endoscópica de la papila de Vater (efectuada por McCune en 1968), ha existido un potente desarrollo tecnológico, que permite realizar el diagnóstico y tratamiento de la patología biliopancreática (Misra \& Dwivedi, 2007).

La CRM que tiene ya establecido un protocolo técnico para su realización permite obtener imágenes precisas de la vía biliar de forma segura y sin riesgos para el paciente. Muchos estudios con series grandes han reportado la capacidad de la CRM para la detección de coledocolitiasis con sensibilidad en rango del 81 al 100\%, especificidad del 85 al $100 \%$, y un diagnóstico preciso en el 89 al $100 \%$ de los casos (Verma et al.; Mogollón Reyes et al., 2014).

La PE es el tratamiento de elección frente a un paciente que presente ictericia obstructiva con perfil bioquímico colestasico y vía biliar dilatada u otro examen imagen que muestre cálculos o tumores obstructivos. Se trata de una cirugía endoscópica, y como tal no exenta de complicaciones (ASGE Standards of Practice Committee; Colton \& Curran; Enns et al.; Howard et al.; Masci et al.).

Se debe destacar en esta serie la alta tasa de canulación exitosa comparable a otras series de centros más grandes. De la misma forma y en concordancia con lo que señala la literatura los resultados muestran la relación existente entre papilotomía y riesgo de hemorragia, no encontrándose asociación de canulacion difícil con pancreatitis posterior al procedimiento (Andriulli et al., 2007; Cotton et al., 2009; Christensen et al., 2004; Freeman et al., 2001; Mallery et al., 2003; Testoni et al., 2010; Wang et al.; Williams et al., 2007).

En una revisión sistemática, basada en 7 ensayos clínicos, que reunen 1039 pacientes, publicada recientemente; y cuyo objetivo era evaluar los resultados en términos de MPO entre uso de pre-cut vs. canulación tradicional, se concluyó que de la MPO es comparable en ambas estrategias; sin embargo el pre-cut no incrementa las complicaciones (hemorragia, perforación y pancreatitis ocurrieron en el $1,8 \% ; 0,4 \%$; y $3,9 \%$ respectivamente de los casos en que se realizó pre-cut group; y en el $0,9 \% ; 0,2 \%$; y $6,1 \%$ respecti- 
vamente cuando se realizó canulación tradicional) (Navaneethan et al., 2014).

Por otra parte, existe evidencia que la frecuencia de pancreatits posterior al procedimientos puede fluctuar entre $5 \%$ y $15 \%$ (Carmes et al., 2006).

Sin embargo llama la atención la alta frecuencia de hemorragia respecto de lo reportado por la literatura (13,5\% vs. 1,8\%) (Colton \& Curran; Ferreira et al., 2007; Losada et al., 2008; Navaneethan et al.), pudiendo deberse a varios factores. Uno de ellos es que la definición de hemorragia es variada (ASGE Standards of Practice Committee) en los di- ferentes estudios, siendo en algunos casos solo significativa si cae el hematocrito o se suspende el procedimiento, definición que de ser aplicada a esta serie, nos daría una frecuencia de sólo $0,5 \%(\mathrm{n}=1)$.

Finalmente y es destacable la alta tasa de canulaciones y la baja tasa de complicaciones observadas en esta serie. Que la canulación difícil no predispone a un mayor riesgo de pancreatitis $(\mathrm{p}=0,519)$; que la hemorragia es la complicación más frecuente observada $13,5 \%(\mathrm{n}=27)$ no demostrándose su asociación con una canulacion difícil $(\mathrm{p}=0,214)$, pero si asociada a la realización de PE $(\mathrm{p}=0,017)$, constituyéndose en un FR.

Figueroa, J.; MANTERola, C.; CASTIllo, E.; FIGUEROA, C.; CARRASCO, R. \& BOHLE, J. Postoperative morbidity by endoscopic retrograde cholangiography with or without ulterior papillotomy. Series of cases. Int. J. Morphol., 33(2):566-570, 2015.

SUMMARY: Bileduct stones is prevalent in our country, with prevalences between $30 \%$ and $50 \%$; and obstructive jaundice secondary to choledocholithiasis (OJC), is a frequent reason of consultation in emergency services. Furthermore, endoscopic papillotomy (EP) post ERCP is the treatment of choice in these cases; however, it is not free of complications (POM). The aim of this study is to describe POM and identify potential risk factors (RF) associated with POM in patients with OJC, underwent PE. Retrospective case series of patients with OJC, who underwent ERCP and subsequent PE. Outcome variable was the development of POM (bleeding, perforation, and pancreatitis). Other variables of interest were cannulation, difficulty of this, developing PE, using precut and mortality. Data collection was performed by an ad-hoc pattern in which the variables extracted from surgical protocols and clinical data were recorded. Descriptive and analytical statistics (Pearson Chi2 and Fisher's exact test) were applied to assess strength of association. 200 patients were operated. The mean age was $60 \pm 18$ years; $62 \%$ were women $(n=124)$. MPO was recorded in 32 cases $(16.0 \%)$ : perforation $(0.5 \%)$, pancreatitis $(2.0 \%)$ and bleeding $(13.5 \%)$. The series does not record mortality. It was not possible to objectify association between "difficult cannulation" and the variables bleeding $(\mathrm{p}=0.214)$; pancreatitis $(\mathrm{p}=0.519)$; and perforation $(\mathrm{p}=1)$. However, association between bleeding and performing PE ( $\mathrm{p}=0.017)$ was observed. Hemorrhage is the most common cause of MPO in this series; and PE is a RF for the development of bleeding.

KEY WORDS: Choledocholithiasis; Choledocholithiasis/surgery; Gallstones; Bile duct diseases; Cholangiopancreatography; Retrograde cholangiopancreatography; Endoscopic retrograde/therapeutic use.

\section{REFERENCIAS BIBLIOGRÁFICAS}

Andriulli, A.; Loperfido, S.; Napolitano, G.; Niro, G.; Valvano, M. R.; Spirito, F.; Pilotto, A. \& Forlano, R. Incidence rates of post-ERCP complications: a systematic survey of prospective studies. Am. J. Gastroenterol., 102(8):1781-8, 2007.

ASGE Standards of Practice Committee; Anderson, M. A.; Fisher, L.; Jain, R.; Evans, J. A.; Appalaneni, V.; Ben-Menachem, T.; Cash, B. D.; Decker, G. A.; Early, D. S.; Fanelli, R. D.; Fisher, D. A.; Fukami, N.; Hwang, J. H.; Ikenberry, S. O.; Jue, T. L.; Khan, K. M.; Krinsky, M. L.; Malpas, P. M.; Maple, J. T.; Sharaf, R. N.; Shergill, A. K. \& Dominitz, J. A. Complications of ERCP. Gastrointest. Endosc., 75(3):467-73. 2012.

Carnes, M.; Cotton, P. B.; Hawes, R. H.; Lawrence, C.; Payne, M. \& Romagnuolo, J. Combined endoscopic biliary sphincterotomy and large balloon dilation for large common bile duct stones. Gastrointest. Endosc., 63(5):AB287, 2006.
Colton, J. B. \& Curran, C. C. Quality indicators, including complications, of ERCP in a community setting: a prospective study. Gastrointest. Endosc., 70(3):457-67, 2009.

Cotton, P. B.; Garrow, D. A.; Gallagher, J. \& Romagnuolo, J. Risk factors for complications after ERCP: a multivariate analysis of 11,497 procedures over 12 years. Gastrointest. Endosc., $70(1): 80-8,2009$.

Christensen, M.; Matzen, P.; Schulze, S. \& Rosenberg J. Complications of ERCP: a prospective study. Gastrointest. Endosc., 60(5):721-31, 2004.

Enns, R.; Eloubeidi, M. A.; Mergener, K.; Jowell, P. S.; Branch, M. S.; Pappas, T. M. \& Baillie, J. ERCP-related perforations: risk factors and management. Endoscopy, 34(4):293-8, 2002. 
Espinel, J.; Pinedo, E. \& Olcoz, J. L. Large hydrostatic balloon for choledocolithiasis. Rev. Esp. Enferm. Dig., 99(1):33-8, 2007.

Ferreira, L. E. \& Baron, T. H. Post-sphincterotomy bleeding: who, what, when, and how. Am. J. Gastroenterol., 102(12):2850-8, 2007.

Freeman, M. L.; Nelson, D. B.; Sherman, S.; Haber, G. B.; Herman, M. E.; Dorsher, P. J.; Moore, J. P.; Fennerty, M. B.; Ryan, M. E.; Shaw, M. J.; Lande, J. D. \& Pheley, A. M. Complications of endoscopic biliary sphincterotomy. N. Engl. J. Med., 335(13):909-18, 1996.

Freeman, M. L.; DiSario, J. A.; Nelson, D. B.; Fennerty, M. B.; Lee, J. G.; Bjorkman, D. J.; Overby, C. S.; Aas, J.; Ryan, M. E.; Bochna, G. S.; Shaw, M. J.; Snady, H. W.; Erickson, R. V.; Moore, J. P. \& Roel, J. P. Risk factors for post-ERCP pancreatitis: a prospective, multicenter study. Gastrointest. Endosc., 54(4):425-34, 2001.

Güitrón-Cantú, A.; Adalid-Martínez, R. \& Gutiérrez-Bermúdez, J. A. Criterios de selección para la realización de colangiopancreatografía endoscópica previo a la colecistectomía laparoscópica. Rev. Gastroenterol. Mex., 67(3):166-70, 2002.

Hermann, R. E. The spectrum of biliary stone disease. Am. J. Surg., 158(3):171-3, 1989.

Howard, T. J.; Tan, T.; Lehman, G. A.; Sherman, S.; Madura, J. A.; Fogel, E.; Swack, M. L. \& Kopecky, K. K. Classification and management of perforations complicating endoscopic sphincterotomy. Surgery, 126(4):658-63, 1999.

Losada, M.; Silva, J. \& Burgos, J. L. Experiencia en colangiografía endoscópica retrógrada en el Hospital Regional de Temuco: Serie de casos conseguimiento. Rev. Chil. Cir., 60(1):5-9, 2008.

Mallery, J. S., Baron, T. H., Dominitz, J. A; Goldstein, J. L.; Hirota, W. K.; Jacobson, B. C.; Leighton, J. A.; Raddawi, H. M.; Varg, J. J. 2nd; Waring, J. P.; Fanelli, R. D.; Wheeler-Harbough, J.; Eisen, G. M.; Faigel, D. O. \& Standards of Practice Committee. American Society for Gastrointestinal Endoscopy. Complications of ERCP. Gastrointest. Endosc., 57(6):633-8, 2003.

Mandelia, A.; Gupta, A. K.; Verma, D. K. \& Sharma, S. The Value of Magnetic Resonance Cholangio-Pancreatography (MRCP) in the Detection of Choledocholithiasis. J. Clin. Diagn. Res., 7(9):1941-5, 2013.

Manterola, C. \& Astudillo, P. Checklist for reporting of descriptive observational studies. MINCIR initiative. Int. J. Morphol., 31(1):115-20, 2013.

Masci, E.; Mariani, A.; Curioni, S. \& Testoni, P. A. Risk factors for pancreatitis following endoscopic retrograde cholangiopancreatography: a meta-analysis. Endoscopy, 35(10):830-4, 2003.
Misra, S. P. \& Dwivedi, M. Is conventional sphincterotomy possible in patients with common bile duct stones impacted at the ampulla of Vater? Trop. Gastroenterol., 28(4):162-5, 2007.

Mogollón Reyes, G.; Sefair, C.; Upegui, D. \& Tovar, C. J. R. Colangiopancreatografía magnética: valor diagnóstico para detectar coledocolitiasis en pacientes con pancreatitis aguda leve. Rev. Cuba. Cir., 53(1):41-51, 2014.

Navaneethan, U.; Konjeti, R.; Venkatesh, P. G.; Sanaka, M. R. \& Parsi, M. A. Early precut sphincterotomy and the risk of endoscopic retrograde cholangiopancreatography related complications: An updated meta-analysis. World J. Gastrointest. Endosc., 6(5):200-8, 2014.

Testoni, P. A.; Mariani, A.; Giussani, A.; Vailati, C.; Masci, E.; Macarri, G.; Ghezzo, L.; Familiari, L.; Giardullo, N.; Mutignani, M.; Lombardi, G.; Talamini, G.; Spadaccini, A.; Briglia, R.; Piazzi, L. \& SEIFRED Group. Risk factors for post-ERCP pancreatitis in high- and low-volume centers and among expert and non-expert operators: a prospective multicenter study. Am. J. Gastroenterol., 105(8):1753-61, 2010.

Verma, D.; Kapadia, A.; Eisen, G. M. \& Adler, D. G. EUS vs MRCP for detection of choledocholithiasis. Gastrointest. Endosc., 64(2):248-54, 2006.

Wang, P.; Li, Z. S.; Liu, F.; Ren, X.; Lu, N. H.; Fan, Z. N.; Huang, Q.; Zhang, X.; He, L. P.; Sun, W. S.; Zhao, Q.; Shi, R. H.; Tian, Z. B.; Li, Y. Q.; Li, W. \& Zhi, F. C. Risk factors for ERCP-related complications: a prospective multicenter study. Am. J. Gastroenterol., 104(1):31-40, 2009.

Williams, E. J.; Taylor, S.; Fairclough, P.; Hamlyn, A.; Logan, R. F.; Martin, D.; Riley, S. A.; Veitch, P.; Wilkinson, M. L.; Williamson, P. R. \& Lombard, M. Risk factors for complication following ERCP; results of a large-scale, prospective multicenter study. Endoscopy, 39(9):793-801, 2007.

\author{
Dirección de Correspondencia: \\ Dr. Julio Figueroa R. \\ Servicio de Cirugía Hospital de Puerto Montt \\ Puerto Montt \\ CHILE
}

Email: drjuliofigueroa@gmail.com

Received: 12-03-2015

Accepted: 22-04-2015 